HNO 2011 · 59:1070-1070

DOI 10.1007/s00106-011-2388-7

Online publiziert: 21. Oktober 2011

(c) Springer-Verlag 2011

\author{
N. Stasche \\ Hals-Nasen-Ohren-Klinik, Schlafmedizinisches Zentrum, \\ Westpfalz-Klinikum, Kaiserslautern
}

\title{
Mittelgesichtsfrakturen im Fokus
}

Mittelgesichtsfrakturen sind im Rahmen von Schädel-Hirn-Traumen häufig. Rohheitsdelikte dominieren vor Verkehrsunfällen und Sportverletzungen als deren Ursachen. Frakturen des Mittelgesichts stellen eine klassische interdisziplinäre Herausforderung dar. Das knöcherne Mittelgesicht beinhaltet mit Sehen, Schmecken und Riechen wichtige Sinnesfunktionen. Zahnhalteapparat, Nasennebenhöhlen und Schädelbasis können in die Verletzungen mit einbezogen sein und lebensbedrohliche intrakranielle Infektionen, schwere Funktionsstörungen des Kauens und Mukozelen der Nebenhöhlen als Spätschäden nach sich ziehen.

Die primäre Diagnostik sollte im Akutfall zunächst die Atemwegssituation klären. Die klinische Untersuchung durch die beteiligten Fachgebiete sollte Fehlstellungen, Instabilität, Hämatome, offene Verletzungen, Liquoraustritt und Funktionsstörungen ebenso einbeziehen wie die Computertomographie-Untersuchung des Gesichtsschädels. Letztere stellt in den unterschiedlichen Schnittrekonstruktionen heute den Goldstandard in der bildgebenden Diagnostik der Mittelgesichtsfrakturen dar.

Die Primärversorgung richtet sich häufig nach regionalen Besonderheiten und der Verfügbarkeit der beteiligten Fachdisziplinen Hals-Nasen-Ohren-Heilkunde (HNO), Mund-Kiefer-Gesichts-Chirurgie (MKG), Neurochirurgie, Ophthalmologie und Intensivmedizin. Der Zeitpunkt und die Reihenfolge der operativen Versorgung ist in den letzten Jahrzehnten viel diskutiert worden. Bei Fehlen vital bedrohender intrakranieller Blutungen haben sich die Prinzipien der
- „Versorgung von innen nach außen“ und das

- „Prinzip der aufgeschobenen Dringlichkeit"

durchgesetzt. Mit der Einführung der Osteosynthese hat sich die Versorgung von Mittelgesichtsfrakturen qualitativ deutlich verbessert. Die primäre belastungsstabile Frakturfixierung führt zu funktionell hervorragenden Ergebnissen. Dies ist unabhängig davon, von welcher Fachdisziplin die Osteosynthese durchgeführt wird. Wichtig ist eine Einbeziehung der beteiligten Fachdisziplinen in allen Phasen der Versorgung. Nur der MKG-Chirurg kann die Okklusion, der HNO-Arzt die Beteiligung von Orbita und Nasenebenhöhlen und der Augenarzt Motilitäts- und Visusstörungen sicher beurteilen.

\section{(? Mit der Osteosynthese hat sich die Versorgung von Mittelgesichtsfrakturen deutlich verbessert}

Eine wichtige Frage bleibt die Entscheidung über die Notwendigkeit der Materialentfernung des Osteosynthesematerials im Mittelgesicht. Die seit vielen Jahren bevorzugten Titanmini- und -mikroplatten haben sich als sehr gut gewebeverträglich erwiesen. Vielerorts werden deshalb Titanplatten beim beschwerdefreien Patienten belassen, auch um durch eine erneute Traumatisierung empfindliche Strukturen wie Orbitainhalt, sensible Trigeminusnervenstrukturen und Tränenwege nicht zu gefährden. In die gleiche Richtung zielte die Entwicklung resorbierbarer Osteosynthesematerialien. Bei ausreichender primärer Materialstabilität stellen sie eine Alternative zu den Titanplatten dar. Über gelegentliche Gewebereaktionen bei der Resorption des Materials, u. a. mit der Gefahr einer Trigeminusreizung, sollte allerdings der Patient ebenso aufgeklärt werden wie über die Vor- und Nachteile der Materialentfernung von Titanplatten.

Mit dem hier vorliegenden Themenheft soll versucht werden, verschiedene Aspekte der Behandlung von Mittelgesichtsfrakturen aus der Sicht verschiedener Fachgebiete anhand der aktuellen Literatur darzustellen. Es soll einen Beitrag zur praxisgerechten, auch interdisziplinären Vorgehensweise bei Mittelgesichtsfrakturen liefern.

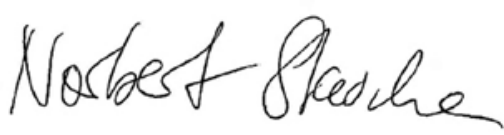

Prof. Dr. N. Stasche

\section{Korrespondenzadresse Prof. Dr. N. Stasche}

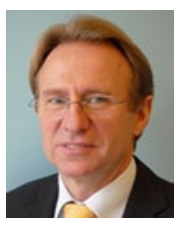

Hals-Nasen-Ohren-Klinik Schlafmedizinisches Zentrum, Westpfalz-Klinikum Hellmut-Hartert-Str. 1, 67655 Kaiserslautern nstasche@westpfalzklinikum.de 